\author{
Military Technical College \\ Kobry El-Kobba \\ Cairo, Egypt
}

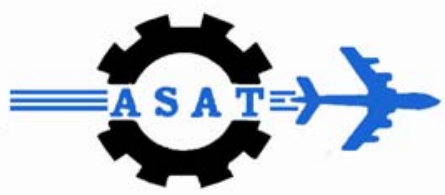

12-th International Conference on

Aerospace Sciences \& Aviation Technology

\title{
DESIGN OF THE GUIDANCE, NAVIGATION AND CONTROL SYSTEM OF A LUNAR LANDER
}

\author{
GUGLIERI* G., PERINO** M.A. and QUAGLIOTTI* F.
}

\begin{abstract}
This paper provides the MDLM (Moon Descent Landing Module) Guidance, Navigation and Control system design description as result of the analysis and trade-off performed in the frame of the "Italian Vision for Moon Exploration" studies of the Italian Space Agency (ASI). The preliminary design of the GNC system for the lunar lander was performed following the analysis of mission and the design requisites, the definition of the RCS architecture, the simulation of system performances, and, finally, the hardware and the equipments.
\end{abstract}

\section{KEY WORDS}

Spacecraft Dynamics, Guidance, Navigation and Control, Space Exploration

\section{NOMENCLATURE}

$\begin{array}{ll}\text { a } & \text { First phase (subscript) } \\ \text { b } & \text { Second phase (subscript) } \\ \text { c } & \text { Third phase (subscript) } \\ \text { D } & \text { Down (subscript) } \\ \text { E } & \text { East (subscript) } \\ \text { f } & \text { Fitness function } \\ \text { GA } & \text { Genetic Algorithm } \\ \text { GNC } & \text { Guidance Navigation and Control } \\ \text { h } & \text { Altitude (m) } \\ I_{S P} & \text { Specific impulse (s) } \\ \text { L } & \text { Landing site (subscript) } \\ \text { MDLM } & \text { Moon Descent Landing Module } \\ m_{P} & \text { Mass of propellant (kg) } \\ m_{0} & \text { MDLM initial mass (kg) } \\ \text { N } & \text { North (subscript) } \\ \text { PID } & \text { Proportional Integrative Derivative }\end{array}$

* Politecnico di Torino, Dipartimento di Ingegneria Aeronautica e Spaziale, Torino, Italy.

** Alcatel Alenia Space, Torino, Italy. 


$\begin{array}{ll}\text { RCS } & \text { Reaction Control System } \\ \mathrm{R} & \text { Slant range }(\mathrm{km}) \\ \mathrm{r}_{1} & \text { Offset - see Fig. } 4(\mathrm{~m}) \\ \mathrm{r}_{2} & \text { Offset - see Fig. } 4(\mathrm{~m}) \\ \text { SOS } & \text { System Of Systems } \\ \mathrm{s} & \text { Range }(\mathrm{km}) \\ \mathrm{T} & \text { Main thrust }(\mathrm{N}) \\ \mathrm{t} & \text { Time }(\mathrm{s}) \\ \mathrm{V} & \text { Velocity }(\mathrm{m} / \mathrm{s}) \\ \alpha_{P} & \text { Thrust-velocity angle (rad) } \\ \gamma & \text { Trajectory angular slope }(\mathrm{rad}) \\ \lambda & \text { Latitude (rad) } \\ \theta & \text { Pitch attitude (rad) } \\ \Omega_{\mathrm{M}} & \text { Planetary angular velocity (rad/s) } \\ 1 & \text { Initial (subscript) } \\ 2 & \text { Final (subscript) }\end{array}$

\section{INTRODUCTION}

After almost 30 years, there is a renewed interest in exploring the Earth's nearest neighbor, the Moon. Recent orbital missions (Clementine and Lunar Prospector) were successfully completed. Surface landing missions are also planned for the next decade with the supervision of space agencies [1][2] even supported by private investors.

Humans and robotic systems visited Earth's natural satellite in the past. During these missions only a small fraction of the Moon's surface was explored and limited samples from those explored areas were returned to Earth. Much remains to be learned about the Moon. As a matter of fact, from what scientists have learned from Apollo [3] and other unpiloted missions, it is known that the Moon may offer resources that could be used in the future to support the exploration activities of the neighboring areas of the solar system.

In the frame of the solar system global exploration, the "Italian Vision for Moon Exploration" represents a basic step to be achieved starting from year 2011 and having the following main objectives:

- Exploration and study of the Moon, including its resources

- Utilization of the Moon as a platform for Universe observation

- Utilization of the Moon as a platform for Earth observation

The "Italian Vision for Moon Exploration" is accomplished by an architecture that globally, for the ground, launch and space segments, foresees several different elements. The strict relationships between the elements and the synergy between their functions shall lead to consider the elements as a complex System Of Systems (SOS). The main elements foreseen by the SOS are:

- the VEGA launcher 
- the Moon ORbiter (MOR)

- the Orbital Transfer Module (OTM)

- the Moon Descent and Landing Module (MDLM)

- the MDLM Payload presently identified as

- an High Mobility Robotic Vehicle (HMRV)

- a generic Moon Surface Element's (MSE)

- a generic Robotic Support System's (RSS)

The current baseline foresees in the launch segment only the VEGA launcher. The VEGA is in charge of the on orbit delivery (LEO) of all the SOS elements and payloads according to the mission scenario that the present study considers more suitable to accomplish the mission objectives. A preliminary description of the current candidate scenario and a related baseline are provided in the next paragraph. The VEGA is launched by the Kourou spaceport and its mission is mainly supported by the ASI and ESA ground centres located both in French Guiana and Europe. In addition to the current operating ground segment, dedicated infrastructure will be considered to support peculiar aspects of the mission like for example the Moon surface operations management.

The Orbital Transfer Module provides the guidance, navigation, control and propulsive capabilities to the Moon Orbiter and to the MDLM (included its payloads) in order to bring them toward the specific Low Lunar Orbit (LLO).

The Moon Orbiter operates in the LLO environment in order to carry out scientific purposes (e.g. lunar surface mapping) and to provide data relay services (e.g. telemetry and telecommands) to the elements operating on the lunar surface, including the MDLM, accordingly to the related designs.

The Moon Descent and Landing Module (MDLM) contributes to the successful achievement of the mission objectives providing to the payloads a safe Moon surface access, then the basic services needed to set the payloads in the proper initial conditions required to start their nominal operations.

The MDLM design involves key technologies for the space exploration. The GNC and propulsion subsystems play an important role in the MDLM design and mission accomplishment together with a high level of system automation and autonomy especially regarding to soft and precision landing phases. In fact, with respect to an interplanetary mission to Mars, the distance between the Earth and Moon introduces only the few seconds in the communications delay; nevertheless such delay does not allow a remote control of the MDLM during the critical maneuvers of landing and obstacle avoidance. As a general remark, the mission statement for the MDLM is safely deliver the payload on moon surface.

The MDLM scenario is based on a single Vega launch whose payload is constituted by the MDLM together with its embedded scientific payload and the MTO that implement 
the electrical propulsion function. The launcher is in charge to put its payloads into a LEO parking orbit from which the MTO, once all the remotely controlled checks from Earth have been resulted positive, can starts its journey to the Moon. This option has been resulted feasible with respect to the insertion of the Vega payload directly into a high elliptic orbit. In fact, the benefit of shorter cruise duration does not compensate the disadvantages coming from the reduction of the Vega payload mass and from the low altitude of the elliptic orbit perigee. In fact, the perigee in this case, being much lower than $400 \mathrm{Km}$, would create intensive atmospheric drag forces on the solar panels then a quick decay of the parking orbit not compensated by the thrust of the solar electrical propulsion.

The cruise is characterized by Earth concentric spirals, typical of the electrical propulsion, and ends with the MTO driving the insertion into the LLO parking orbit. The LLO orbit is a stable configuration during which all the checks of the MDLM, still attached to the MTO, are conducted to verify the system status before the separation.

The entry and descent phase has been modeled into two steps: a Hohmann transfer from the $100 \times 100 \mathrm{~km}$ parking orbit to a lower orbit $18 \times 18 \mathrm{~km}(\mathrm{DV}=38 \mathrm{~m} / \mathrm{s})$ then a propelled free fall that is almost vertical in the very last part $(D V=1700 \mathrm{~m} / \mathrm{s})$. The lander utilizes two control techniques: modulation of both main thrust (pulsed thrusters) and attitude/directional thrusters. While some redundancy exists, the optimum operating conditions will use each technique where best suited. In any case, the lander takes full advantage of its propulsion system for control.

Although some tolerance for the landing site accuracy is accepted ( $1 \mathrm{~km} \times 1 \mathrm{~km})$, there is still a need for GNC. Correct landing attitude and descent rate are two of these needs. Landing attitude will be predominately controlled by RCS while descent rate is controlled by thrusting power. Obstacle avoidance and landing site terminal correction are provided by RCS thrusters. The onboard vision-based system is also linked with GNC for landing site correction (the image pattern recognition works as an outer loop closure for trajectory corrections).

Even if the design of GNC systems for lunar landers is detailed in several concept studies and future mission proposals [4][5][6][7][8] available from the review of current technical publications and reports, the experience of Apollo is still a unique reference for the designer [9][10][11][12]. In the present study the design of the GNC system for the lunar lander was analyzed following a sequence of steps: definition of hardware and equipments, analysis of mission and design requisites, sizing of RCS subsystem, simulation and optimization of mission performances. The preliminary results of this analysis will be presented and discussed in the following sections.

\section{THE GNC SYSTEM}

The reference descent profile (see Fig.1) is shortly described to clarify the sequence of actions performed by the GNC system. The descent of the MDLM on the Moon will be accomplished autonomously by the spacecraft. The vehicle will be oriented along a 
North-South axis during landing. The lander will begin its descent with the engine aligned with the direction of orbit $(18 \times 18 \mathrm{~km})$. The main thrusters will fire at maximum thrust level during descent according to a prescribed sequence, with rotational adjustments and minor course corrections being accomplished with the RCS thrusters. The descent will be initially programmed using position data obtained from examination of outer view (sun angle sensors and Moon horizon sensors) and lunar surface imagery. The onboard IMU will be used continuously during descent as the primary mean of tracking spacecraft location. At approximately $1 \mathrm{~km}$ in altitude (high gate), the altimeter(s) will obtain lock on the lunar surface and be able to provide the exact distance from the surface. As velocity is lost, the spacecraft will rotate with vertical alignment with no horizontal velocity and controlled vertical descent rate for the very last phase (low gate at $\mathrm{h}=100 \mathrm{~m}$ ). The onboard vision-based system will examine the landing site for hazards (e.g. rocks and deep craters) and have the opportunity to modify the exact landing location acting on the RCS. The impact with the lunar surface is expected to be soft $(\mathrm{V} \approx 0 \mathrm{~m} / \mathrm{s})$.

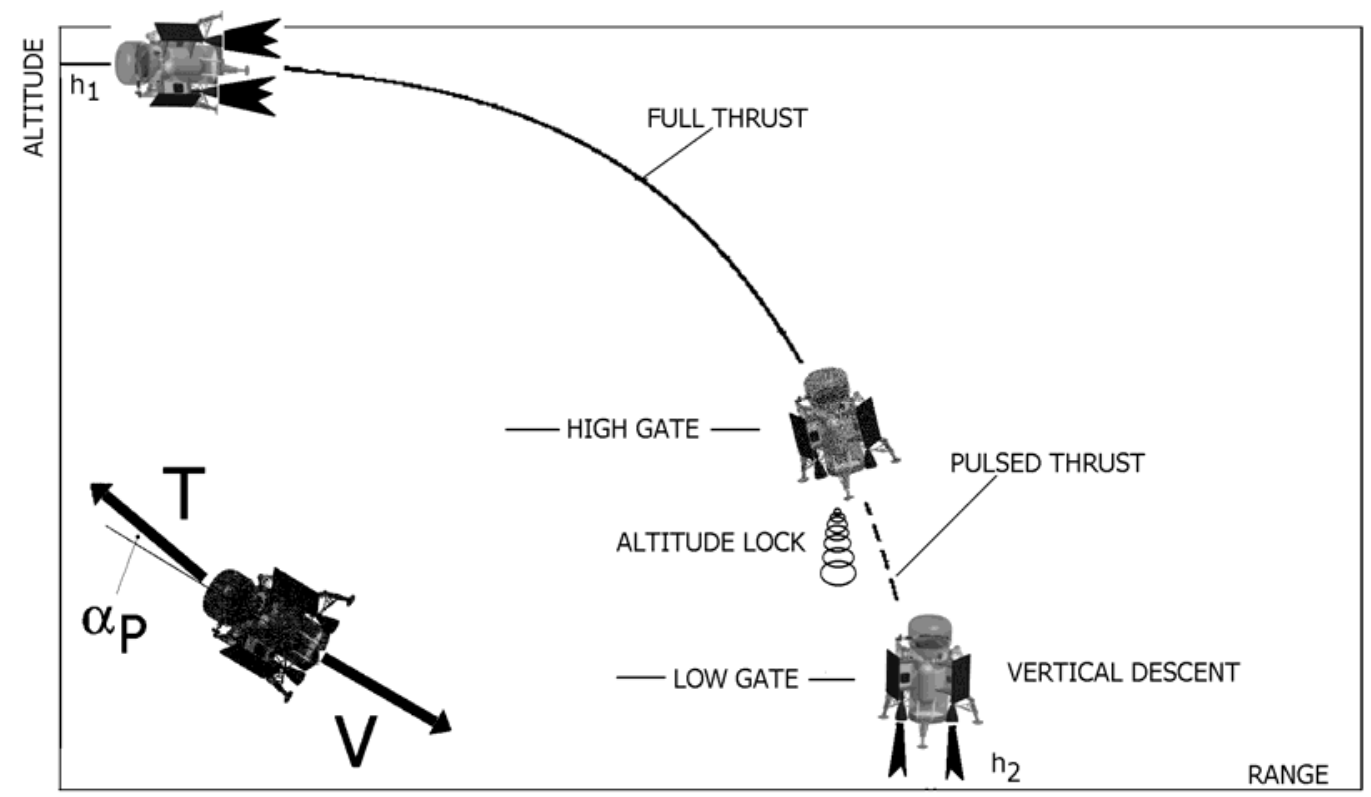

Fig.1. Phases of the lunar landing.

The GNC system functional logics are presented in Fig.2. Guidance software first compute the lander location required to satisfy mission requirements, navigation sensors and algorithms then track the vehicle's actual location, and flight control then transports the MDLM to the required location.

The elaborations required by the GNC system are performed by a PC board. Multitasking should ensure the redundancy of the most critical software tasks.

Navigation is based on the acquisitions of 2 IMU units digitally filtered with data available from other sensors: 
- Sun and horizon sensors during the complete descent profile after MTO separation $(\mathrm{h}<100 \mathrm{~km})$

- radar altimeter during the vertical descent $(\mathrm{h}<1000 \mathrm{~m})$

- vision system during the vertical descent $(\mathrm{h}<1000 \mathrm{~m})$

- laser altimeter during the last part of the vertical descent $(\mathrm{h}<100 \mathrm{~m})$.

All sensors are interfaced to the elaboration unit via standard serial protocol. The numerical integration of inertial data is based on an accurate gravitational model corrected for local altitude and position.

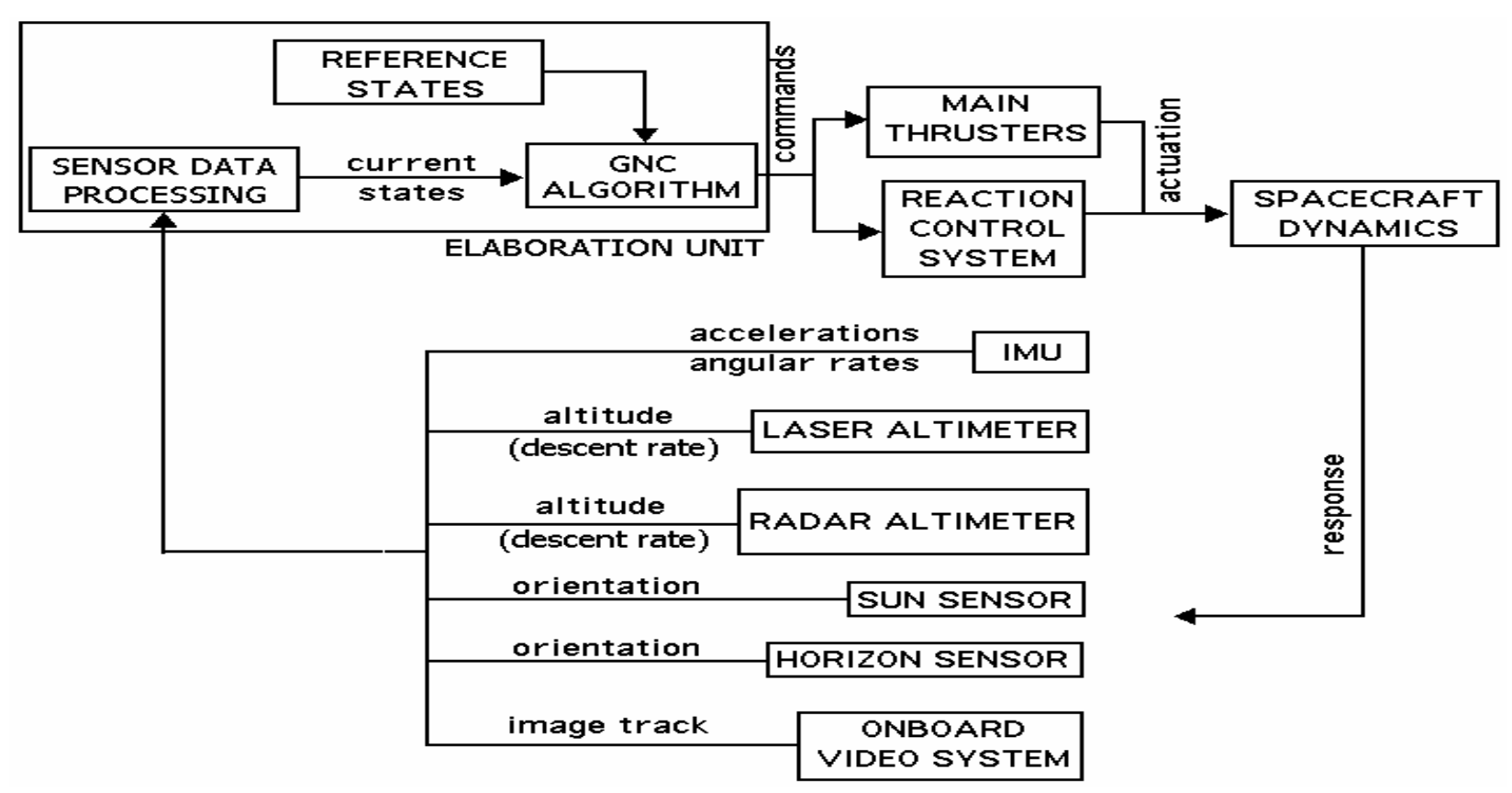

Fig.2. Schematic functional layout of the GNC system.

The reference inertial measurement accuracy (after data processing) is $\pm 1 \mathrm{deg} / \mathrm{h}$ for angular rates and $\pm 0.025 \mathrm{~m} / \mathrm{s}^{2}\left(2.5 \cdot 10^{-3} \mathrm{~g}\right)$ for accelerations. During the initial part of the descent the accuracy of trajectory tracking must ensure that angles and velocities are estimated with an adequate margin ( $\pm 1 \mathrm{mrad}$ for angles and $\pm 2.5 \mathrm{~m} / \mathrm{s}$ for velocities) in order to obtain the required target accuracy $(1 \mathrm{~km} \times 1 \mathrm{~km})$. The insertion of altimeters in the measurement loop is expected to enhance the accuracy of trajectory estimation during the critical phases prior to landing $( \pm 0.25 \mathrm{~m} / \mathrm{s})$. The use of the vision system should also improve the target accuracy acting on the outer guidance loop.

The guidance algorithm is based on a programmed sequence of desired states derived from a reference descent profile. Desired attitude scheduling enforces the alignment of the thrust axis with the nominal descent trajectory as a consequence of RCS control actions. The variable used for guidance scheduling is the radial distance from target landing location (i.e. vehicle's actual range and altitude). Adequate resistance to thrust asymmetries and torque damping about the inertial axis of the lander is provided by the 
RCS that should react with a conventional PID loop closure (inner loop). The error in the feedback control is given by the difference between actual and desired attitudes (pitch, roll and yaw angles). The action of main thrusters is programmed as a minimal sequence of full thrust shoots that should decelerate the vehicle from orbital speed to terminal vertical descent. During this very last phase $(\mathrm{h}<100 \mathrm{~m})$ an outer primary feedback loop is enabled where both RCS and main thrusters are triggered by measured altitude and descent rate as the lander should follow the nominal touch down profile. The vision system acts on the RCS with a separate secondary outer loop for obstacle avoidance and landing site alignment.

For the present analysis the GNC system was implemented as closed-loop control acting on RCS (see Fig. 5) and main thrusters according to the scheduled descent profile. Sensors were simplified (without digital filters) and measurement errors were assumed to be constant parameters superimposed to simulation outputs.

\section{THE REFERENCE MODEL}

Trade-off for design parameters was performed using a specific software simulation tool. The mathematical model of the lander was developed for off-line dynamics analysis. It is a nonlinear representation of a rigid vehicle. The effects of main thrusters and controls (RCS thrusters) are superimposed in terms of force increments. The rigid body motion of the spacecraft is modeled using six nonlinear force and moment equations while the usual three kinematic relations (Euler equations) are replaced by quaternions. The most important feature of these equations of motion is that the states need not to be small quantities; thus, all the kinematic nonlinearities associated with the motion of the rigid body are retained.

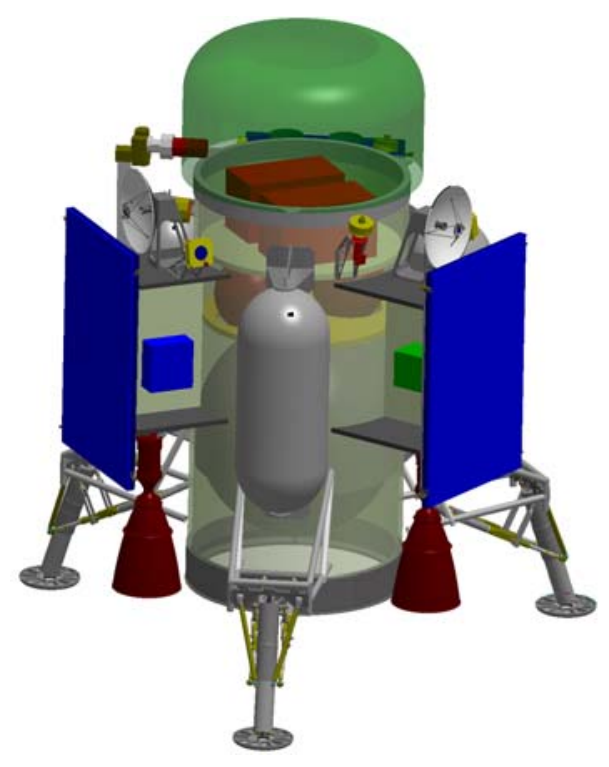

Fig.3. External view of MDLM. 
The model of the gravitational field of the Moon was corrected accounting for altitude. The gravitational components are included in the dynamic model.

The equations of spacecraft rotational dynamics were implemented assuming principal body axes while external forces (gravitational components and main thrust) acting on the vehicle were reduced in NED frame. Planetary effects on body accelerations (transport, centripetal and Coriolis accelerations) were also modeled, including latitude and longitude reconstruction during the descent.

The total initial mass $m_{0}$ of the lander $\left(h_{1}=18 \mathrm{~km}\right.$ initial altitude) is $1350 \mathrm{~kg}$ including propellant $\left(m_{P}=600 \mathrm{~kg}\right.$ and $\left.m_{P} / m_{0}=0.45\right)$. The inertial properties (mass and moments of inertia) are supposed to vary with time as a function of mass decrease due to fuel consumption. The lander is modeled with an equivalent cylinder (diameter $2.5 \mathrm{~m}$ and height $2 \mathrm{~m}$ ) with uniform mass distribution (see Fig. 3 for reference). The shift of center of gravity due to mass reduction is considered as fuel tanks are located below the nominal center of mass of the spacecraft. Moments of inertia are updated accordingly.

The following characteristics for the main thrusters are used as a reference:

- 3 symmetrically located engines

- MMH-MON propellant

- Nominal specific impulse $I_{\mathrm{sp}}=317 \mathrm{~s}$

- Total maximum thrust $<8 \mathrm{kN}$

- Pulsed control of main thrust (50 ms minimum active thrust time interval)

- Thrust feedback on descent rate during vertical descent

- Thrust is aligned with the axis of symmetry of the vehicle (normal operation)

The Reaction Control System has two separate functions: platform attitude control and trajectory accurate tracking. The system is based on the following architecture:

- A primary set of 8 thrusters

- A secondary set of 8 thrusters for redundancy (not considered)

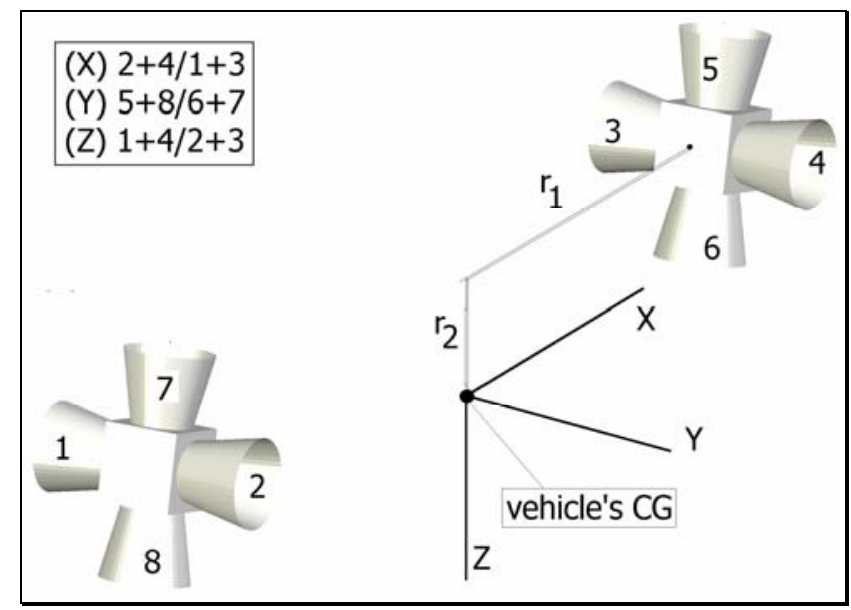

Fig.4. Reaction Control System (layout). 
The action of primary RCS thrusters is expected to provide a torque correction as neutral as possible in terms of transverse forces. The control torques are produced as a response to angular displacements from nominal reference attitudes and angular rates measured by the onboard inertial sensors (PID controller). The thrusters are mounted along the sides of the lander (see Fig. 4). For the present analysis, the offset distances are assumed to be $r_{1}=1.1 \mathrm{~m}$ and $r_{2}=0.8 \mathrm{~m}$. As the trajectory is aligned with the $X-Z$ body plane, the rotation about the $X$ axis is used for out-of-plane corrections. The descent attitude of the vehicle is controlled by the $Y$ rotation. A secondary set of thrusters should act in parallel for redundancy. The design is conducted assuming that this secondary attitude control system is inactive (worst case analysis). The thrusters of the RCS are limited to $20 \mathrm{~N}$ (maximum thrust). The same MMH-MON propellant is used but the nominal specific impulse is reduced to $I_{s p}=285 \mathrm{~s}(10 \%$ decrease taking into account the losses of the thrusters). Pulsed operating mode is considered and the RCS is supposed to have less than 20 ms minimum active thrust time interval.

The control logics were implemented according to the functional sequence described in Fig. 5 in which the thrusters of RCS were assumed to operate in pulsed mode.

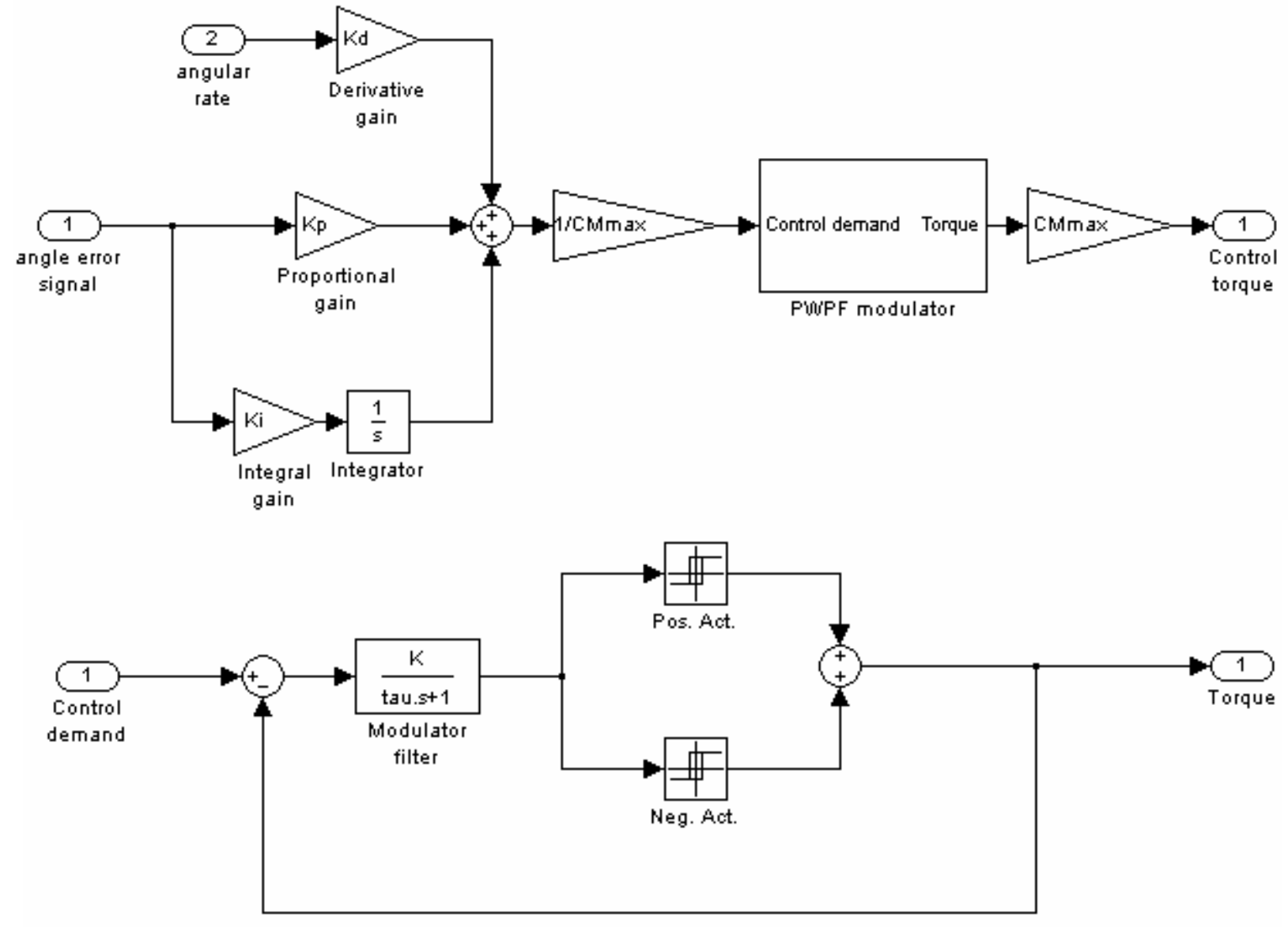

Fig.5. Reaction Control System (numerical model). 


\section{RESULTS}

The preliminary trajectory analysis was performed with a reduced order model in which the rotational dynamics of the vehicle was neglected, the RCS was disabled and the thrust was assumed to be tangent to the trajectory. The primary aim of this analysis is the sizing of the propulsion system based on the starting altitude $h_{1}=18 \mathrm{~km}$ and the definition of adequate landing site altitude margins. Continuous full thrust was considered during the descent. The impact of thrust levels on trajectory profiles is given in Fig. 6 in which the range for deceleration is limited within the range $T / \mathrm{m}_{0}=1.5 \div 6 \mathrm{~m} / \mathrm{s}^{2}$ equivalent to $T=2 \div 8 \mathrm{kN}$. As the thrust is increased the North range $s_{N}$ decreases substantially reducing the time required to land. The final altitude $\mathrm{h}_{2}$ to complete speed reduction $(\mathrm{V}=0 \mathrm{~m} / \mathrm{s})$ increases with the deceleration $\mathrm{T} / \mathrm{m}_{0}$ provided by main thrusters.

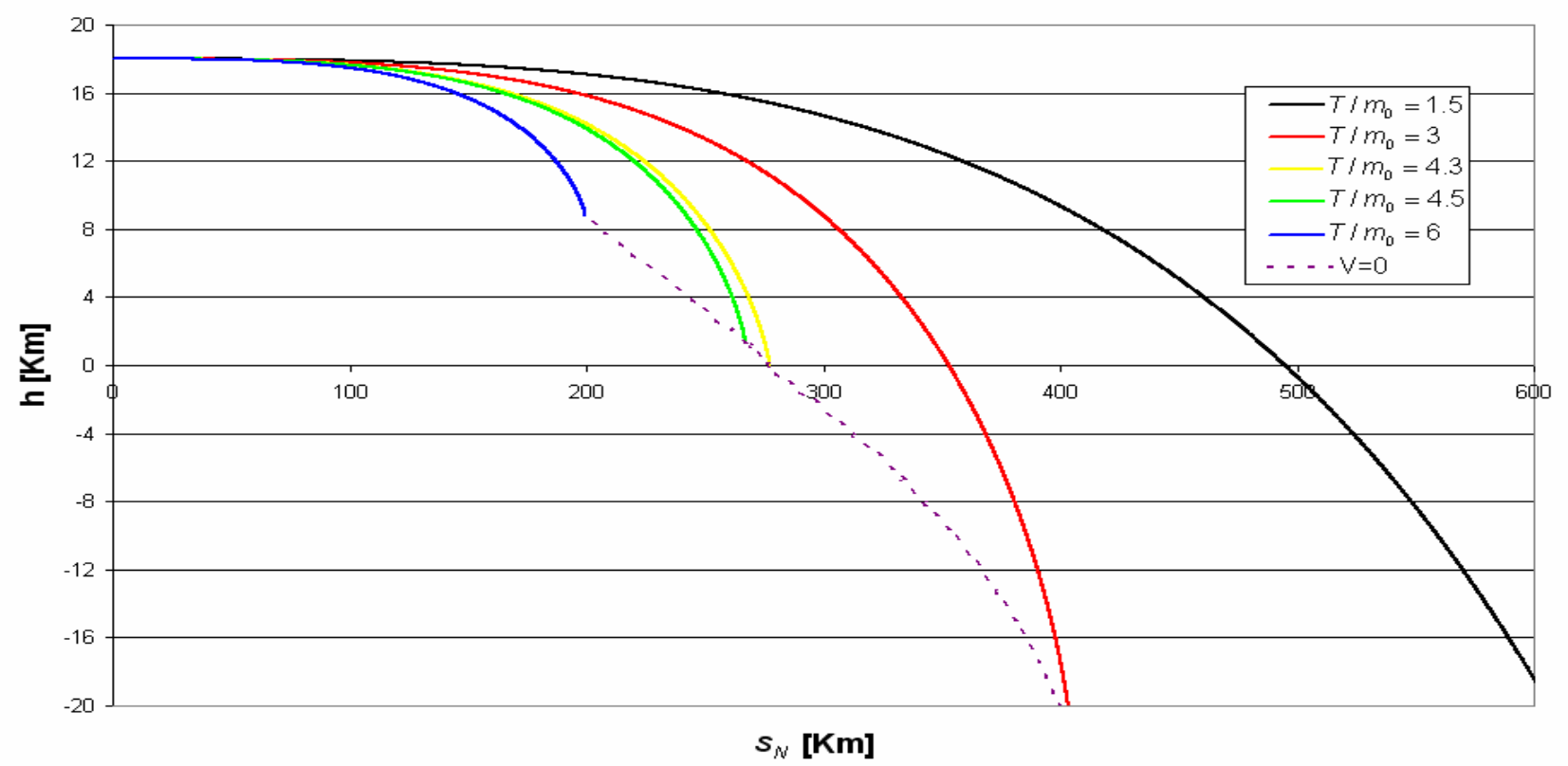

Fig.6. Effect of thrust levels on descent profiles (trajectory).

The diagram also demonstrates that $h_{2}=h_{L}=0 \mathrm{~m}$ can be obtained for $T / \mathrm{m}_{0}=4.3 \mathrm{~m} / \mathrm{s}^{2}$ $\left(T=5.8 \mathrm{kN}\right.$, range $\mathrm{S}_{N}=280 \mathrm{~km}$ and time to land $t_{2}=312 \mathrm{~s}$ ) while landing site altitude $h_{L}$ can be increased up to $9000 \mathrm{~m}$ by providing the maximum deceleration rate $\left(T / \mathrm{m}_{0}=6 \mathrm{~m} / \mathrm{s}^{2}\right.$ equivalent to $\mathrm{T}=8 \mathrm{kN}$ ). Deceleration profiles are reproduced in Fig. 7. The diagram shows that the effect of mass reduction on the deceleration rate during the descent is limited as it remains below $10 \mathrm{~m} / \mathrm{s}^{2}$. As expected, the deceleration increases with landing site altitude.

The fuel fraction $m_{P} / m_{0}$ required to completely decelerate the vehicle $(\Delta V=1700 \mathrm{~m} / \mathrm{s})$ is presented in Fig. 8 as a function of thrust levels. The results show that the reference fuel fraction $\left(m_{P} / m_{0}=0.45\right)$ is adequate for $T / m_{0}>3 \mathrm{~m} / \mathrm{s}^{2}(T=4 \mathrm{kN})$ with a residual fuel margin $\left(\Delta \mathrm{m}_{\mathrm{P}} / \mathrm{m}_{0}=0.02 \div 0.025\right.$ equivalent to $\left.\Delta \mathrm{m}_{\mathrm{P}}=30 \div 35 \mathrm{~kg}\right)$. 


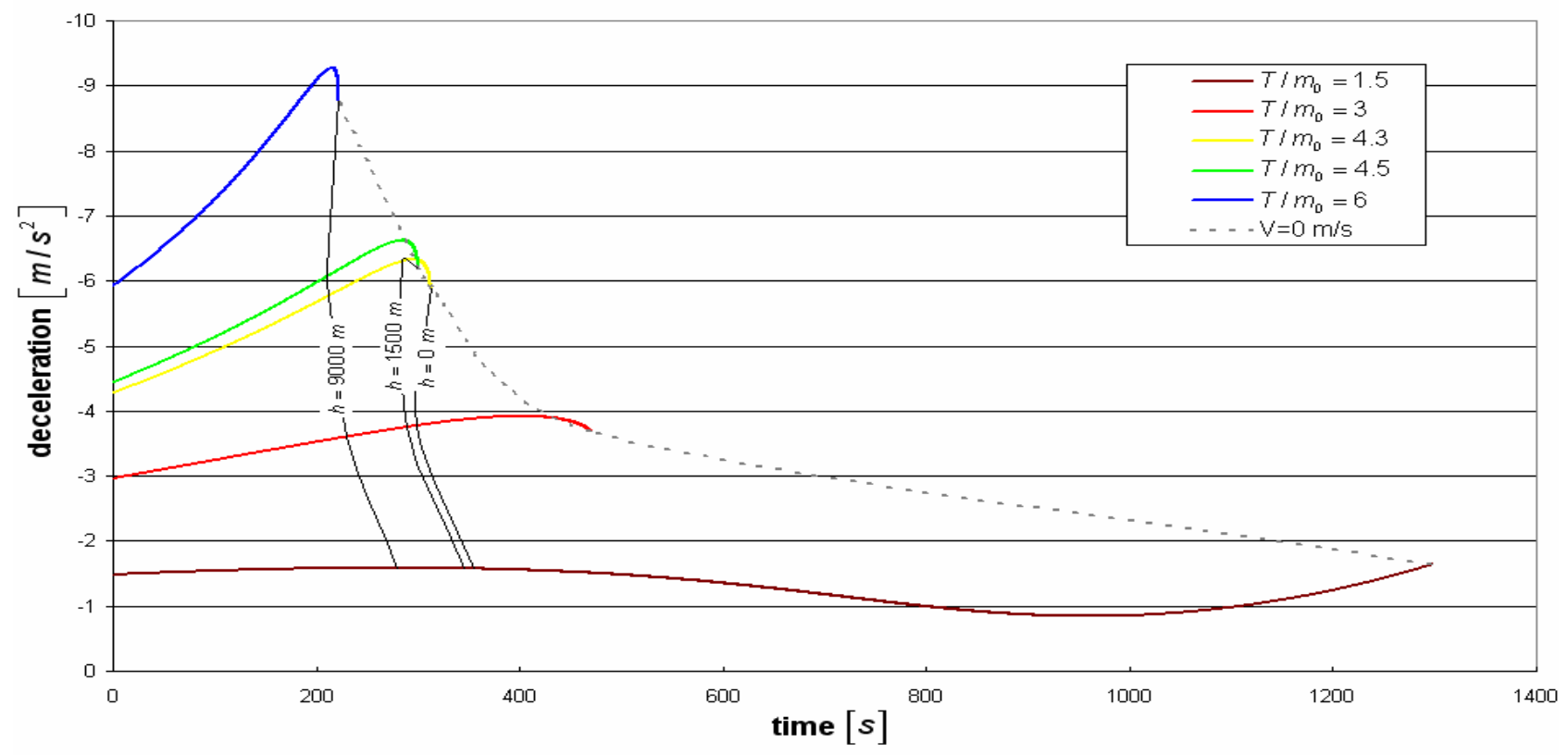

Fig.7. Effect of thrust levels on descent profiles (deceleration).

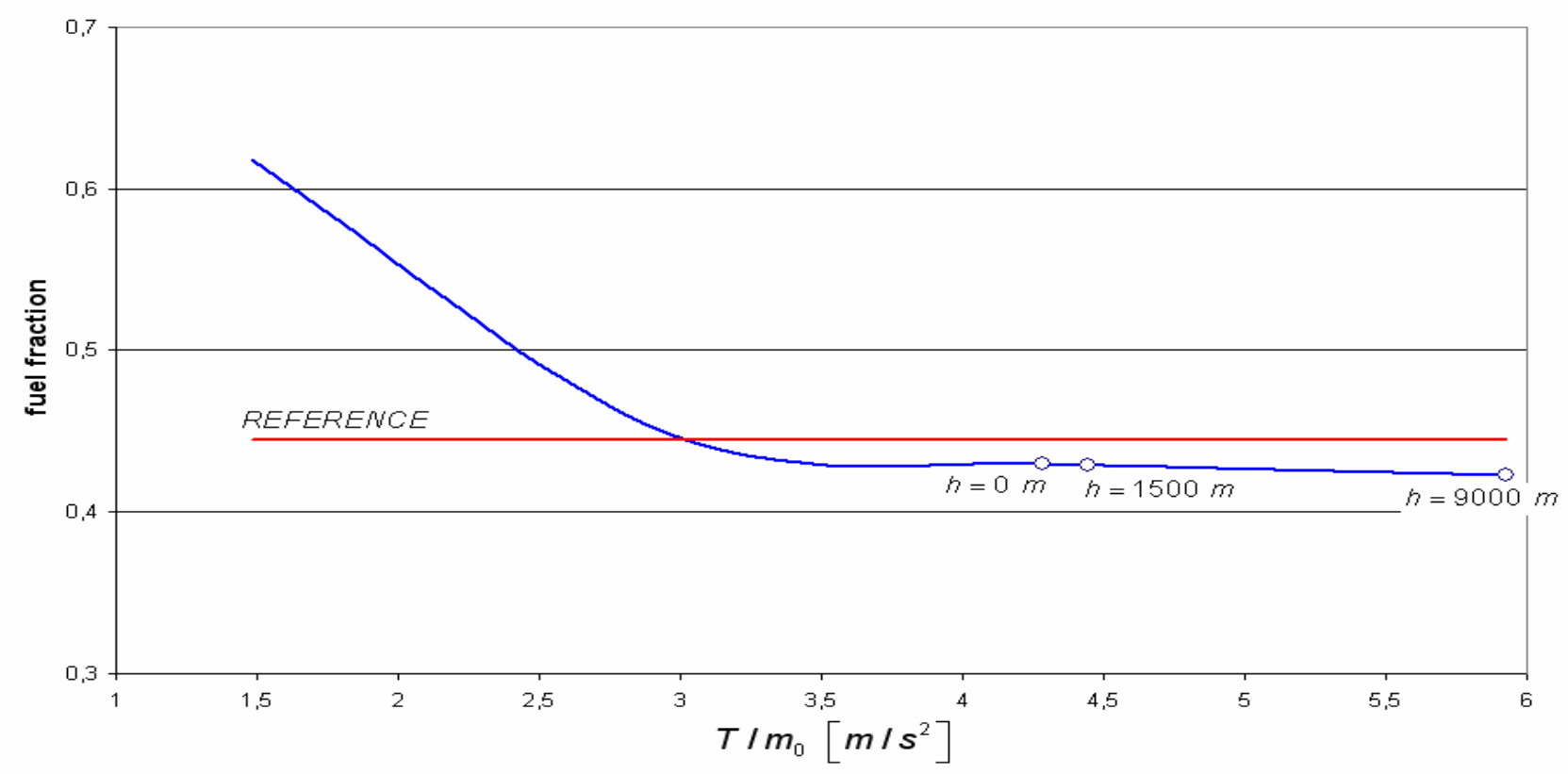

Fig.8. Effect of thrust levels on descent profiles (mass).

The preliminary analysis also demonstrated that the impact of landing site latitude $\lambda$ on the sidewise trajectory deviation induced by the Coriolis acceleration term $-2 \cdot \Omega_{\mathrm{M}} \cdot\left(\mathrm{V}_{\mathrm{N}}\right.$ $\left.\cdot \sin (\lambda)+V_{D} \cdot \cos (\lambda)\right)$ is limited to $1 \mathrm{~km}$ (worst case polar landing starting from $\lambda=-80^{\circ}$ and targeting approximately $\left.\lambda=-90^{\circ}\right)$. This deviation disappears as the landing target approaches equatorial sites and it becomes minimum also for shorter trajectories. Nevertheless, equatorial landing sites are affected by target shift due to lunar rotation occurring during the descent. 
The preliminary analysis is concluded considering the effect of thrust-velocity alignment for constant thrust descent $\left(T / \mathrm{m}_{0}=3 \mathrm{~m} / \mathrm{s}^{2}\right.$ and $\left.\mathrm{T}=4 \mathrm{kN}\right)$. The effect is reproduced in Fig. 9 where the main thrust (the direction opposites the flames) is aligned $\alpha_{P}$ degrees above the anti-velocity vector. The results show that trajectory control can be enhanced using an appropriate scheduling of $\alpha_{P}$ (typically for lunar descent $\alpha_{P}=5^{\circ} \div 10^{\circ}$ ) with an acceptable impact on fuel mass budget. As a general concern, matching of final deceleration altitude $(V=0)$ with landing site $\left(h_{2}=h_{L}\right)$ for low thrust profiles is obtained with a substantial increase of range that should be minimized (sidewise deviation effects).

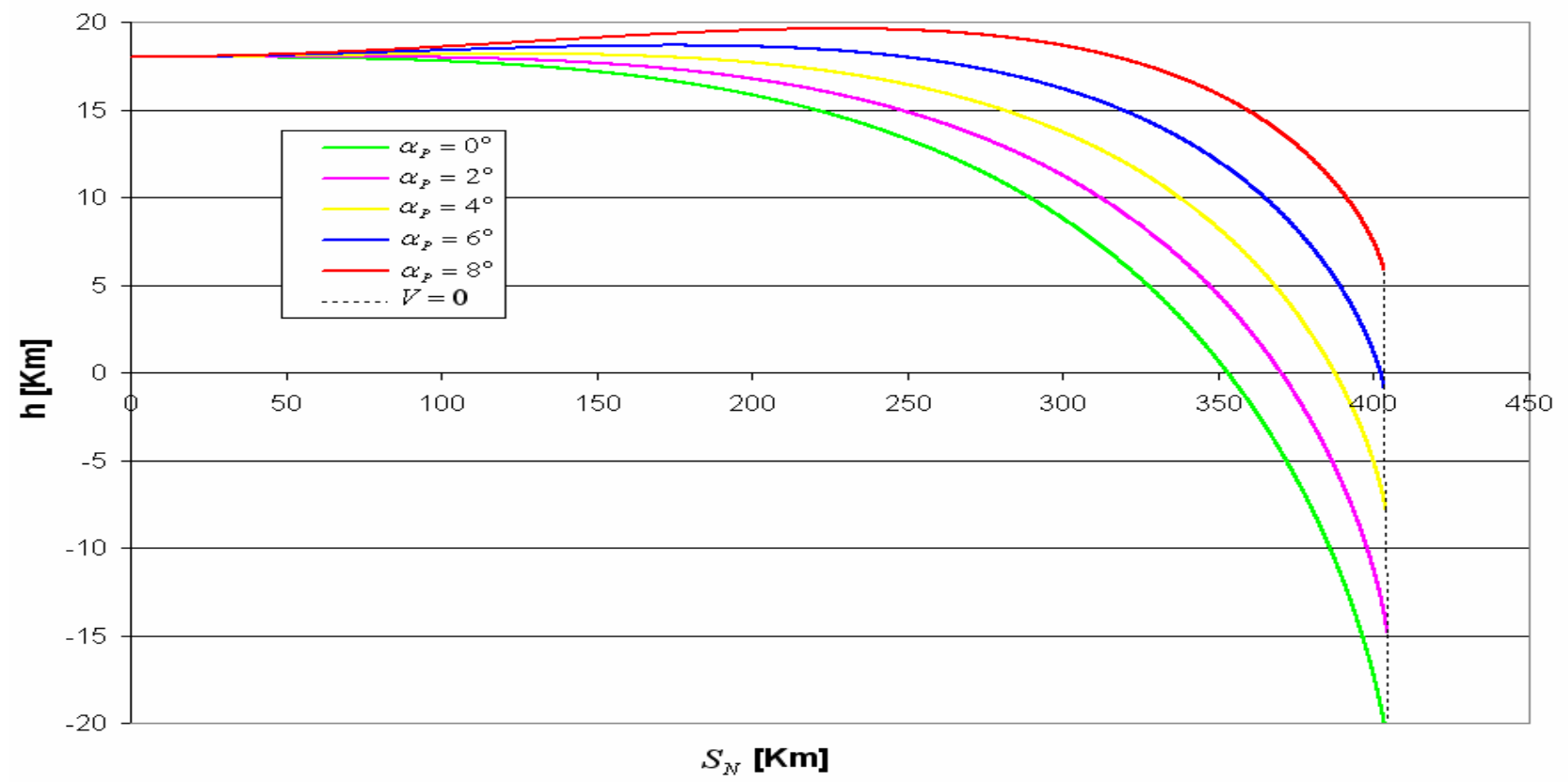

Fig.9. Effect of thrust-velocity alignment on descent profiles (trajectory).

The trajectory profiles (based on the reduced order model) were also optimized so as to meet the requirements for terminal vertical descent $\left(h_{2}=h_{L}, V_{2}=0\right.$ and $\left.\gamma_{2}=90^{\circ}\right)$. The solver adopted for the design of the descent trajectory (i.e. to obtain the scheduling of the main thrusters) is a Fortran version of the genetic driver described by D. Carroll in [13]. The code initializes a random sample of individuals with different parameters to be optimized using the genetic algorithm (GA) approach. The convergence to solution was reached enabling the micro genetic switch for the search algorithm. This last option significantly reduces the number of function evaluations with a faster convergence to the nearoptimal region. Very briefly, a micro-GA starts with a random, very small population. The population evolves in normal GA fashion and converges in a few generations. Then, a new random population is chosen while keeping the best individual from the previously converged generation and the evolution process restarts. Note that average population fitness values are not meaningful with a micro-GA because of the start-restart nature of the micro-GA evolution process. The code was set for a maximum micro population size of 5 individuals, 60 bits per individual and a maximum of 5 parameters (i.e. 12 binary bits per parameter and $2^{12}$ possible solutions per parameter). The fitness function $f$ to be 
optimized is supplied by means of an external subroutine called by the solver. In this case, the fitness function was designed in order to meet the requirements for touchdown:

$$
f=\frac{1}{\left(h_{2}-h_{L}\right)^{2}+V_{2}^{2}+\left(\gamma_{2}-\pi / 2\right)^{2}}
$$

Numerical experiments were performed dividing the trajectory of the lander into active propulsive segments with different thrust levels. As a matter of fact, a constant propulsive deceleration provides inadequate fitness in terms of terminal slope $\gamma_{2}$. Finally, after some tuning of the algorithm, the landing sequence was split in 3 phases assuming $\alpha_{P}=0^{\circ}$ : a) full thrust deceleration with $\left.T_{a}=8 \mathrm{kN}, b\right)$ descent without deceleration with $T_{b}=0$ and c) final descent with $T_{c}<8 \mathrm{kN}$ (throttable or pulsed thrust). The unknowns of the optimization procedure are the thrust level $T_{c}$ and the time intervals $t_{a}$ and $t_{b}$ i.e. each individual in the search process is characterized by 3 parameters. The results are summarized in Tab. 1 for different landing altitudes $h_{L}$.

The results show that the optimization process identifies some solutions characterized by shorter range $s_{N}$ and faster landing (reduction of time $t_{2}$ ) when compared with the constant thrust descent profile. The duration $t_{b}$ of the inactive segment is minimized by the algorithm (less than $15 \%$ of the landing time $t_{2}$ ) and the terminal conditions respect the design requirements $\left(V_{2}=0\right.$ and $\gamma_{2}=93^{\circ}$ for $\left.h_{L}=0\right)$. As a remark, some deceleration is still required in the very last part of the descent trajectory $\left(V=73 \mathrm{~m} / \mathrm{s}\right.$ and $\gamma=25^{\circ}$ for $\mathrm{h}=1000 \mathrm{~m}$ while $\mathrm{V}=29 \mathrm{~m} / \mathrm{s}$ and $\gamma=39^{\circ}$ for $\mathrm{h}=100 \mathrm{~m}$ ). Furthermore, the slope of the trajectory is still far from the vertical reference for $h=100 \mathrm{~m}$. This means that the vertical descent phase cannot be included in the present formulation of the optimization procedure as significant actions of the RCS should control this part of the landing sequence.

\begin{tabular}{|c|c|c|c|c|c|c|c|c|c|c|}
\hline $\mathrm{h}_{\mathrm{L}}(\mathrm{m})$ & $\mathrm{t}_{\mathrm{a}}(\mathrm{s})$ & $\mathrm{T}_{\mathrm{a}}(\mathrm{kN})$ & $\mathrm{t}_{\mathrm{b}}(\mathrm{s})$ & $\mathrm{T}_{\mathrm{b}}(\mathrm{kN})$ & $\mathrm{h}_{\mathrm{c}}-\mathrm{h}_{2}(\mathrm{~m})$ & $\mathrm{t}_{\mathrm{c}}(\mathrm{s})$ & $\mathrm{T}_{\mathrm{c}}(\mathrm{kN})$ & $\mathrm{h}_{2}(\mathrm{~m})$ & $\mathrm{t}_{2}(\mathrm{~s})$ & $\mathrm{s}_{\mathrm{N}}(\mathrm{km})$ \\
\hline 0 & 108 & 8.00 & 41 & 0.00 & 13000 & 140 & 6.67 & 0 & 289 & 251 \\
+4000 & 186 & 8.00 & 14 & 0.00 & 5600 & 76 & 4.35 & +4000 & 276 & 210 \\
-4000 & 154 & 8.00 & 15 & 0.00 & 15800 & 160 & 3.84 & -4000 & 329 & 232 \\
\hline
\end{tabular}

Table 1. Effect of landing site altitude on optimal descent profiles (90000 generations).

Hence, the GA will be used to address the design of the descent for the MDLM from $h_{1}=18 \mathrm{~km}$ to $h_{2}=100 \mathrm{~m}$ with $V_{2}=10 \mathrm{~m} / \mathrm{s}$ and $\gamma_{2}=90^{\circ}$ keeping $\alpha_{P}=0^{\circ}$. 


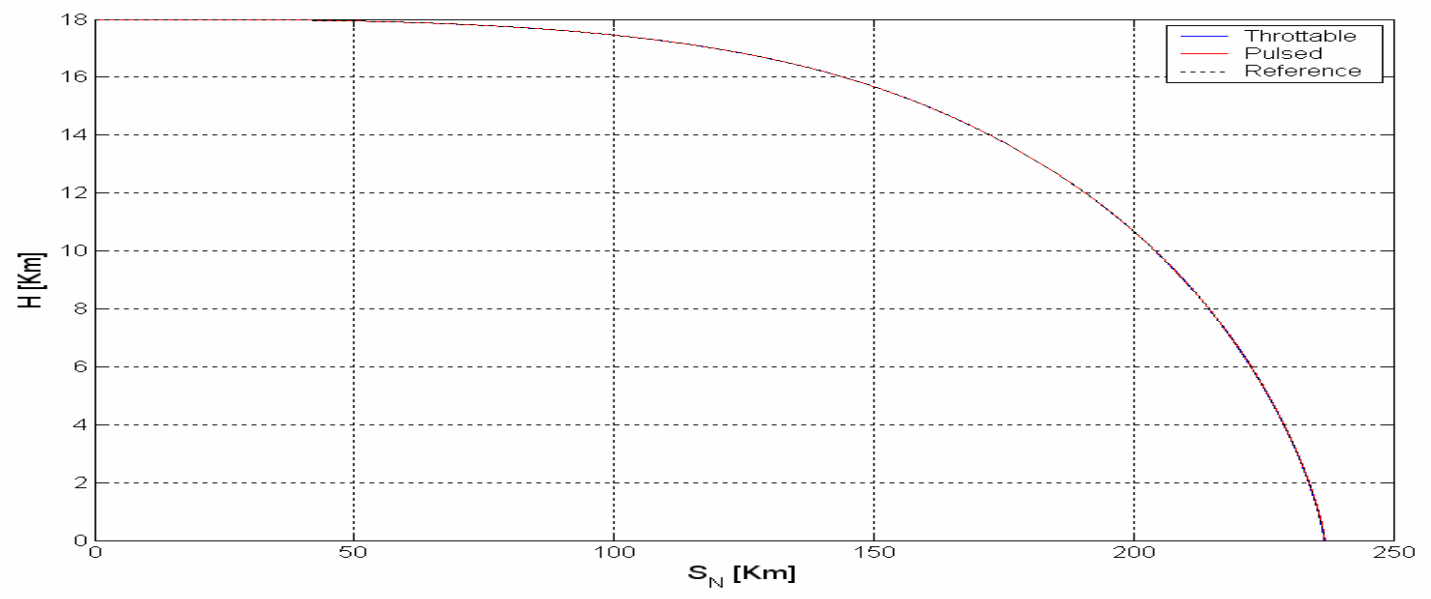

Fig.10. Effect of thrust control mode on descent profiles (range).

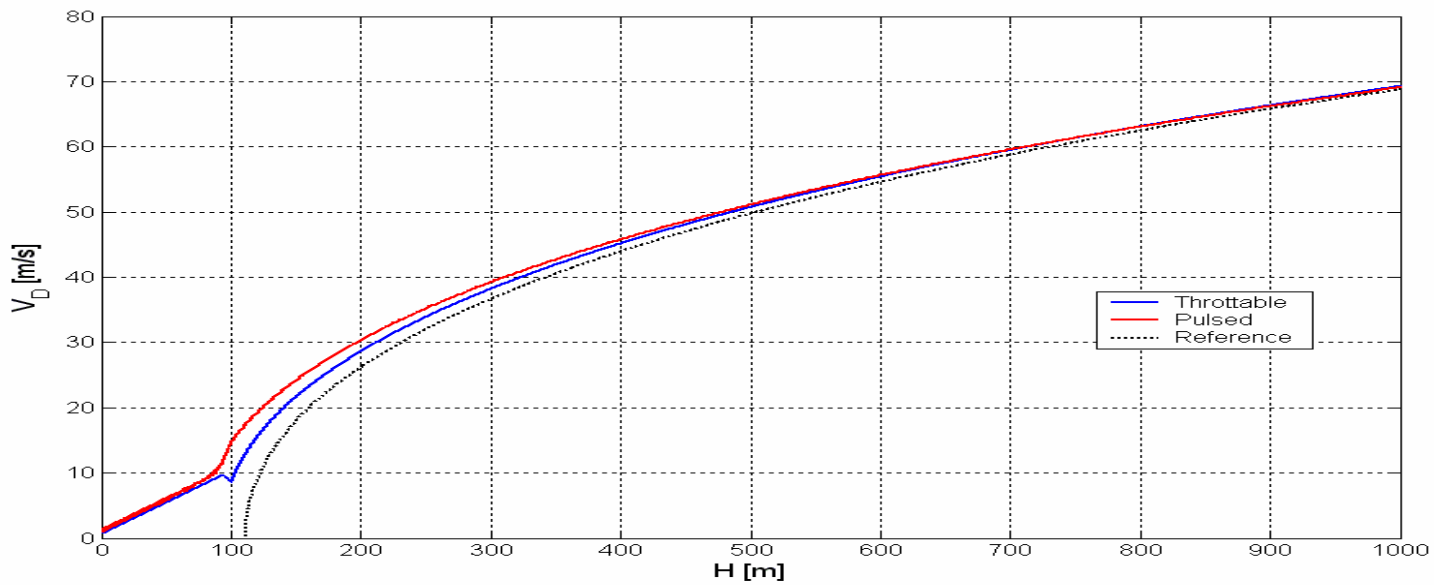

Fig.11. Effect of thrust control mode on descent profiles (velocity).

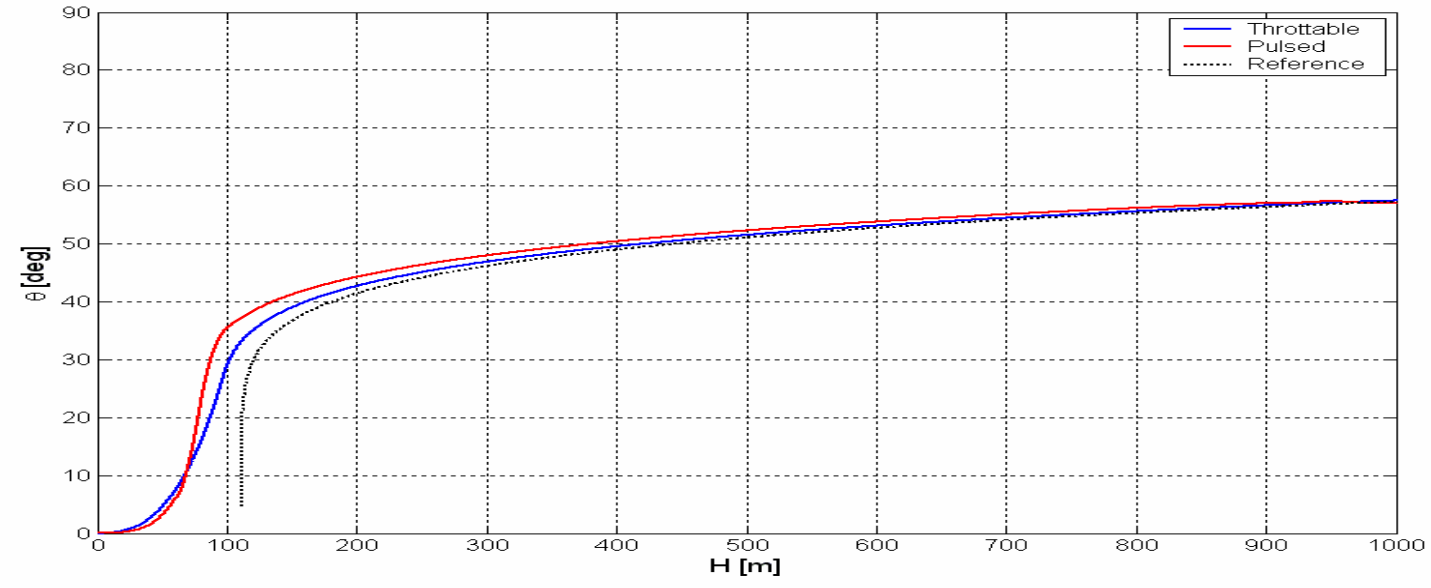

Fig.12. Effect of thrust control mode on descent profiles (pitch angle). 
Detailed simulations with a higher order model of the dynamic system will provide adequate support for the design of the terminal descent phase. Another concern will be the altitude lock made by the radar altimeter that will be performed for non-vertical attitudes of the landing module $\left(\gamma \neq 90^{\circ}\right.$ for $\left.h=1000 \mathrm{~m}\right)$ : an active tilt control of the antenna will be required.

The analysis of the complete landing sequence is carried out with simulations including attitude dynamics. The optimal thrust solution (3-phase deceleration obtained with the GA optimization) is used for the design of the guidance sequence. The main thrust level and the reference attitudes of the vehicle are scheduled according to the slant range distance

$$
R=\sqrt{\left(s_{N}-s_{N_{2}}\right)^{2}+\left(h-h_{2}\right)^{2}}
$$

The distance $R$ is obtained assuming that the aiming point is located at $h_{2}=100 \mathrm{~m}$ above the landing site location (low gate altitude). When the lander crosses the high gate altitude $(\mathrm{h}=1000 \mathrm{~m})$ thrust and attitudes are speed-scheduled according with $\mathrm{V}_{\mathrm{N}}$ while the constant deceleration profile for the final descent below the low gate altitude $(h=100$ $\mathrm{m}$ ) is scheduled with $\mathrm{V}_{\mathrm{D}}$. The coordinates of landing site are corrected for lunar rotation occurring during the descent. The update frequency of the guidance algorithm for the reference attitudes and thrust outputs is set to $100 \mathrm{~Hz}(0.01 \mathrm{~s})$ while the numerical integration is performed with a reduced time step for the pulsed system (0.005 s).
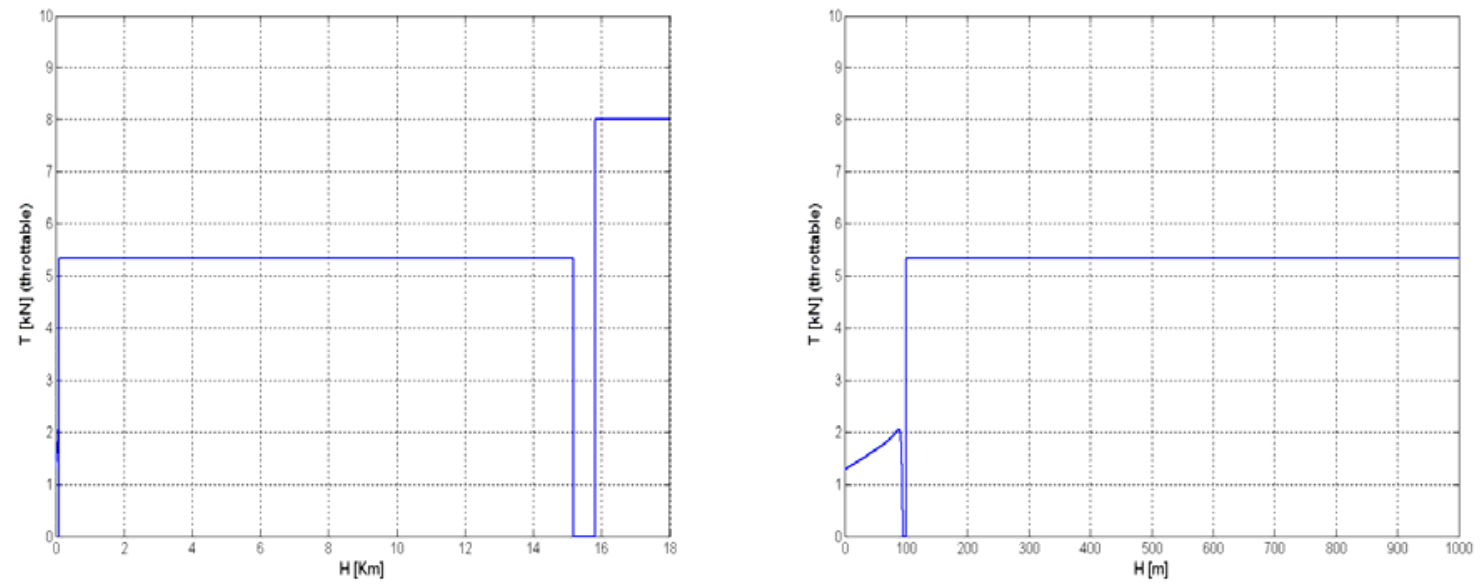

Fig.13. Effect of thrust control mode on descent profiles (main thrust).

The results of simulations are presented in Fig. 10, 11, 12 and 13 assuming polar landing starting from $\lambda=-90^{\circ}$ and targeting approximately $\lambda=-80^{\circ}$. Three strategies are compared: nominal 3-phase deceleration (point mass model), 3-phase deceleration with either throttable or pulsed thrusters including attitude dynamics and RCS control. In any case, out of plane trajectory deviation is low $(0.15 \mathrm{~km})$ kept within the limits of RCS steering corrections. The gains of the feedback control on vehicle's attitudes were set close to the upper limits for closed-loop stability. The RCS actions were checked for thrust time interval (acceptable when lower than $20 \mathrm{~ms}$ ) and perturbation limits 
simulated with the superposition of a time-dependent inertial load (fuel sloshing). The impact of attitude control on fuel consumption is minimal (less than $2 \mathrm{~kg}$ ) for the two controlled descents. The very last vertical descent phase (the lower gate is fixed at $\mathrm{h}=100 \mathrm{~m}$ ) is completed in less than $30 \mathrm{~s}$ with an accurate velocity control performed by the main thrusters. This last phase was found to be critical when operated with pulsed thrusters as very short shoots $(50 \div 100 \mathrm{~ms})$ are required to obtain a precisely controlled descent and a soft touch-down. This stringent requisite in terms of bandwidth of the propulsion system can be probably relaxed increasing the low gate altitude $(\mathrm{h}>>100 \mathrm{~m}$ ) with a larger tolerance on terminal attitudes of the lander. Numerical experiments also show that the introduction of a no-thrust rapid rotation phase just before crossing the lower gate altitude could be beneficial (accurate vertical alignment).

\section{CONCLUDING REMARKS}

The preliminary design of the GNC system for a lunar lander was completed following the analysis of mission requisites, the definition of the RCS architecture, the simulation of system performances, and, finally, the hardware and the equipments. The propulsion system and the fuel fraction were sized with a trade-off analysis based on a simplified constant thrust descent profile. Altitude margins and deceleration envelopes were verified. An optimization procedure based on a genetic algorithm was used to design the descent trajectory and the scheduling of the main thrusters. The simulations performed indicate that the response of the system is also adequate in terms of target precision and attitude dynamics. The RCS thrusters were checked to minimize the offset between actual and reference descent profile. A preliminary characterization in terms of architecture and accuracy of GNC hardware was finally introduced. Future work will be addressed to the refinement of mission analysis including the definition of optimal $\alpha_{P}$ and main thrust minimization. A complete mathematical model of sensors (including statistical errors) will also be mandatory for the assessment of GNC details.

\section{ACKNOWLEDGEMENTS}

The present research activity was supported by Alcatel Alenia Space as prime contractor for the MDLM design in the frame of the "Italian Vision for Moon Exploration" studies of the Italian Space Agency (ASI).

\section{REFERENCES}

[1] http://www.esa.int, (ILEWG) International Lunar Exploration Working Group, (2007).

[2] http://nssdc.gsfc.nasa.gov/planetary, National Space Science Data Center, NASA Goddard Space Flight Center, USA, (2007).

[3] "Remembering Apollo 11 The 35th Anniversary Data Archive CD-ROM", NASA History Office, Washington DC, USA, (2004). 
[4] Kassing, D., Novara, M., "Lunar European Demonstration Approach - A First Step in ESA's Lunar Exploration Initiative", ESA-ESRIN Bulletin No. 82, (1995).

[5] “EuroMoon 2000 Mission Summary”, ESA-ESRIN BR-122, (1996).

[6] http://www.tsgc.utexas.edu, Archives of the Texas Space Grant Consortium, the University of Texas at Austin, USA.

[7] Head, J.N., Hoppa, G.V., Gardner, T.G., Seybold, K.S., Svitek, T., "Autonomous Low Cost Precision Lander For Lunar Exploration", 36th Lunar and Planetary Science Conference, USA, (2005)

[8] Loucks, M., Carrico, J., Carrico, T., Deiterich, C., "A Comparison Of Lunar Landing Trajectory Strategies Using Numerical Simulations", International Lunar Conference, Canada, (2005).

[9] Bennett, F.V., "Apollo Lunar Descent and Ascent Trajectories", NASA TM-X-58040, USA, (1970).

[10] Bennett, F.V., "Apollo Experience Report - Mission Planning for Lunar Module Descent and Ascent", NASA TND-6846, USA, (1972).

[11] Klumpp, A.R., "Apollo Lunar Descent Guidance", Massachusetts Institute of Technology, R-695, USA, (1971).

[12] Eyles, D., "Tales From The Lunar Module Guidance Computer", 27th AAS Guidance and Control Conference, USA, (2004).

[13] Carroll, D.L., "Chemical Laser Modeling With Genetic Algorithms", AIAA Journal, Vol. 34, No. 2, pp 338-346, (1996). 